\title{
Oliguria without serum creatinine increase after living donor liver transplantation is associated with adverse post-operative outcomes
}

\section{$\operatorname{AUTHOR}(\mathrm{S})$ :}

Mizota, T.; Minamisawa, S.; Imanaka, Y.; Fukuda, K.

\section{CITATION:}

Mizota, T.... [et al]. Oliguria without serum creatinine increase after living donor liver transplantation is associated with adverse post-operative outcomes. Acta Anaesthesiologica Scandinavica 2016, 60(7): 874-881

\section{ISSUE DATE:}

2016-08

URL:

http://hdl.handle.net/2433/254487

\section{RIGHT:}

This is the peer reviewed version of the following article: Mizota, T, Minamisawa, S, Imanaka, Y, Fukuda, K. Oliguria without serum creatinine increase after living donor liver transplantation is associated with adverse post - operative outcomes. Acta Anaesthesiologica Scandinavica 2016 Aug;60(7):874-81, which has been published in final form at https://doi.org/10.1111/aas.12722. This article may be used for non-commercial purposes in accordance with Wiley Terms and Conditions for Use of Self-Archived Versions.; この論文は出版社版でありません。引用の際には出版社版を ご確認ご利用ください。; This is not the published version. Please cite only the published version. 
Oliguria without serum creatinine increase after living-donor liver transplantation is associated with adverse postoperative outcomes

Toshiyuki Mizota $^{1, *}$, Satoshi Minamisawa ${ }^{2}$, Yuichi Imanaka ${ }^{2}$, Kazuhiko Fukuda $^{1}$

${ }^{1}$ Department of Anaesthesia, Kyoto University Hospital, 54 Shogoin-Kawahara-cho, Sakyo-ku, Kyoto 606-8507, Japan

${ }^{2}$ Department of Healthcare Economics and Quality Management, Graduate School of Medicine, Kyoto University, Yoshida Konoe-cho, Sakyo-ku, Kyoto 606-8501, Japan

Short title: Oliguria after Liver Transplantation

Word count excluding abstract and references: 2,491

* Corresponding author: Toshiyuki Mizota

Assistant Professor

Department of Anesthesia, Kyoto University Hospital, 54 Shogoin-Kawahara-Cho, Sakyo-Ku, Kyoto 606-8507, Japan

Tel: +81-75-751-3433; Fax: +81-75-752-3259

Email:mizota@kuhp.kyoto-u.ac.jp 


\begin{abstract}
Background: Acute kidney injury (AKI) is a common complication after liver transplantation and is associated with significant morbidity and mortality. Although clinical guidelines recommend defining AKI based on serum creatinine increase and oliguria, the validity and utility of the oliguric component of AKI definition remains largely unexplored. This study examined the incidence and the impact on clinical outcomes of oliguria meeting the urine output criterion of AKI in patients undergoing liver transplantation. The authors hypothesized that oliguria was an independent risk factor for adverse postoperative outcomes.
\end{abstract}

Methods: This study retrospectively examined 320 patients who underwent living-donor liver transplantation at our centre. AKI stages were allocated according to recent guidelines based on serum creatinine or urine output within 7 days of surgery.

Results: The incidence of oliguria meeting the urine output criterion of AKI was 50.3\%. Compared with creatinine criterion alone, incorporating oliguria into the diagnostic criteria dramatically increased the measured incidence of AKI from $39.7 \%$ to $62.2 \%$. Compared with patients diagnosed without AKI using either criterion, oliguric patients without serum creatinine increase had significantly longer intensive care unit stays (median: 5 vs. 4 days, $\mathrm{P}=0.016$ ), longer hospital stays (median: 60 vs. 49 days, $\mathrm{P}=0.014$ ) and lower chronic kidney disease-free survival rate on postoperative day $90(54.2 \%$ vs. $73.3 \%, \mathrm{P}=0.008)$.

Conclusion: Oliguria is common after liver transplantation, and incorporating oliguria into the diagnostic criteria dramatically increases the measured incidence of AKI. Oliguria without serum creatinine increase was significantly associated with adverse postoperative outcomes. 


\section{Introduction}

Acute kidney injury (AKI) is a common complication after liver transplantation and is associated with significant morbidity and mortality ${ }^{1-3}$. Although several studies have analysed the incidence, risk factors and outcomes of AKI after liver transplantation, multiple AKI definitions made it difficult to compare results. Therefore, researchers and clinicians must have a consensus for defining AKI.

To standardise the AKI definition, the risk, injury, failure, loss of function and end-stage renal failure guideline criteria were developed ${ }^{4}$. These were subsequently revised by the Acute Kidney Injury Network ${ }^{5}$ and the Kidney Disease Improving Global Outcomes (KDIGO) group ${ }^{6}$. Although these definitions involve increases in serum creatinine $(\mathrm{SCr})$ and oliguria to diagnose $\mathrm{AKI}$, in practice, the urine output criterion of AKI definition is frequently discarded because of difficulties in making consistent measurements ${ }^{7,8}$.

While some studies have investigated the role of urine output criterion in defining $\mathrm{AKI}^{9-13}$, the validity and utility of the oliguric component of AKI definition remains largely unexplored. Within the context of liver transplantation, previous studies did not explore the incidence or clinical significance of oliguria. Therefore, this study examined the incidence and impact on clinical outcomes of oliguria meeting the urine output criterion of AKI in patients undergoing liver transplantation. We hypothesized that oliguria was an independent risk factor in this patient group for worsened outcomes. 


\section{Methods}

This study was approved by the ethics committee of the Kyoto University Hospital (approval number: R0060, 22 Jun, 2015), and written informed consent was waived. We analysed the medical records of adult ( $\geq 18$ years old) patients who underwent living-donor liver transplantation (LDLT) at the Kyoto University Hospital from January 2006 to December 2014. Patients with fulminant hepatic failure were excluded. In addition, patients who had end-stage renal disease preoperatively [i.e. estimated glomerular filtration rate (eGFR) $<15 \mathrm{~mL} / \mathrm{min} / \mathrm{m}^{2}$ as determined using a formula validated in Japan: eGFR $=194 \times \mathrm{SCr}^{-1.094} \times \mathrm{age}^{-0.287} \times 0.739$ (if female) ${ }^{14}$ or receipt of long-term haemodialysis] were also excluded because assessment of AKI was not relevant.

Patients were divided into five categories according to the indication of LDLT: hepatocellular carcinoma, hepatitis $\mathrm{B} / \mathrm{C}$ cirrhosis, alcoholic cirrhosis, cholestasic liver disease (primary biliary cirrhosis or primary sclerosing cholangitis) and others. The following patient and operative variables were also collected: patient characteristics, Model for End-stage Liver Disease (MELD) score ${ }^{15}$, Child-Pugh classification, preoperative SCr, previous liver transplantation, blood type compatibility, graft-recipient weight ratio, intraoperative blood loss, amount of intraoperatively used blood products, intraoperative urine output and operative time. $\mathrm{SCr}$ concentration was measured using LABOSPECT 008 (Hitachi High-Technologies, Tokyo, Japan).

AKI was diagnosed and staged according to recent guidelines ${ }^{6}$ (Table 1). Data from daily SCr measurement within 7 days of surgery and urine output measured every 2 hours during postoperative intensive care unit (ICU) stay (within 7 days of surgery, in case postoperative ICU stay was $>7$ days) were used to assign AKI stages. Preoperative SCr value was considered as a baseline value. We allocated AKI stages based on SCr criterion (AKI-Cr) and urine output criterion (AKI-UO), respectively; then the higher stage of AKI-Cr or AKI-UO was used for staging according to the standard AKI definition (AKI-com).

The basic immunosuppressive regimen consisted of calcineurin inhibitor (tacrolimus or cyclosporine) and low-dose corticosteroids ${ }^{16}$. Patients who received blood-type-incompatible 
transplants had preoperative anti-CD20 antibody (rituximab $375 \mathrm{mg} / \mathrm{m}^{2}$ ) with preoperative plasma exchange, postoperative hepatic artery infusion of prostaglandin E1 $(0.01 \mu \mathrm{gg} / \mathrm{kg} / \mathrm{min})$ and methylprednisolone (125 mg/day), as well as postoperative cyclophosphamide $(2 \mathrm{mg} / \mathrm{kg} / \mathrm{day})$ followed by mycophenolate mofetil (starting dose, $500 \mathrm{mg} /$ day; maintenance dose, $1000 \mathrm{mg} /$ day) ${ }^{17-19}$. Adverse outcomes for analysis included all cause hospital mortality, time to ICU and hospital discharge, 90-day mortality and chronic kidney disease (CKD)-free survival (the proportion of patients who are alive and free of CKD) at 90 days after LDLT. CKD was defined as an eGFR of $<60 \mathrm{~mL} / \mathrm{min} / 1.73 \mathrm{~m}^{2}$ at 90 days after LDLT according to the criteria established by the National Kidney Foundation's Kidney Disease Outcomes Quality Initiative, which defined CKD as a glomerular filtration rate of $<60 \mathrm{~mL} / \mathrm{min} / 1.73 \mathrm{~m}^{2}$ for $>3$ months $^{20}$.

\section{Statistical analyses}

Data were analysed using the statistical program R (http://cran.r-project.org). The data are presented as median (interquartile range) and number (percentage), unless otherwise stated. Differences between groups were compared using the Mann-Whitney U test for continuous variables. For categorical variables, the Pearson chi-square test or Fisher exact test was used where appropriate. Time-to-event analyses were used to compare lengths of ICU and hospital stays. Patient data were censored at the time of death. Medians and interquartile range were obtained using the Kaplan-Meier analyses, and the log-rank test was used to assess differences between groups. Simple and multivariable logistic regression analyses were used to assess the impact of AKI-Cr or AKI-com on hospital mortality. In the multivariable analysis, adjustments were made for previously described risk factors (recipient age, MELD score $>30$, re-transplantation and blood-type incompatibility) ${ }^{21,22}$. We calculated the odds ratios of AKI-Cr or AKI-com; and then those of each stage of AKI-Cr or AKI-com. The areas under the receiver operating characteristic curves (AUC-ROC) were calculated to assess the ability of AKI-Cr or AKI-com to predict hospital mortality. All statistical tests were 
two-tailed and statistical significance was set at $\mathrm{P}<0.05$ (with Dunnett correction for multiple comparisons yields a $\mathrm{P}$ value of $<0.017$ ). 


\section{Results}

Altogether, 373 adult patients underwent LDLT during the study period. Of these, 320 eligible patients were analysed after excluding 21 patients with fulminant hepatic failure, 31 patients who had end-stage renal disease preoperatively, and one patient whose data concerning urine output in ICU were missing. The ages of the patients ranged from 18 to 69 years, and 154 patients (48.1\%) were female. 203 patients $(63.4 \%)$ received diuretics [furosemide $(0.8-4 \mathrm{mg} / \mathrm{h})$ or human atrial natriuretic peptide (hANP, 0.06-0.3 mg/h)] during postoperative ICU stays; $88(27.5 \%)$ received hANP only, $57(17.8 \%)$ received furosemide only, and 58 (18.1\%) received both. Additionally, 256 patients $(80.0 \%)$ were administered dopamine during ICU stays.

\section{Incidences of AKI by diagnostic criteria}

Altogether, AKI-Cr occurred in 127 patients (39.7\%), whereas AKI-UO was diagnosed in 161 patients $(50.3 \%), 89(55.3 \%)$ of whom also met the criterion for AKI-Cr (Fig. 1). Combining oliguric or SCr-based criteria to define AKI as per KDIGO guidelines, 199 patients (62.2\%) were diagnosed as AKI; the AKI incidence increased about 1.6 times compared to that using SCr-based criterion alone. Table 2 shows the patient characteristics and perioperative variables stratified by the AKI status. The graft-recipient weight ratio was lower, and intraoperative blood loss and volume of blood transfused were higher in patients with both AKI-Cr and AKI-UO compared with patients diagnosed without AKI using either criterion. Patients with AKI-Cr only or those with AKI-UO only were comparable in demographics and operative variables to patients without AKI, except that more female patients were included in AKI-UO only group and this group tended to suffer from more blood loss. Most patients who developed AKI-UO received diuretics (furosemide or hANP) during ICU stays. Table 3 shows the association between the AKI status and distribution of AKI stages according to the standard AKI definition (AKI-com). More than half of patients with AKI-Cr only or AKI-UO only were allocated to stage 1 AKI according to the standard AKI definition; in contrast, most patients (73.0\%) with both AKI-Cr and AKI-UO were allocated to stage 2-3 AKI. 
Clinical outcomes according to AKI status

Overall, patients with both AKI-Cr and AKI-UO had a significant increase in hospital and 90-day mortality, prolonged ICU or hospital stays, and lower CKD-free survival rate at postoperative day (POD) 90. Neither AKI-Cr only nor AKI-UO only were significantly associated with an increase in hospital or 90-day mortality, but patients with AKI-UO only had longer ICU or hospital stays and lower CKD-free survival rate at POD 90 (Table 4).

To examine whether incorporating oliguria into the diagnostic criteria for AKI improved predictive ability, we compared the performance of AKI-Cr and AKI-com staging in predicting hospital mortality. After adjustment for known predictive factors (age, MELD score $>30$, blood-type incompatibility and re-transplantation), both AKI-Cr and AKI-com were significantly associated with an increase in hospital mortality [AKI-Cr: odds ratio (OR) 2.07, 95\% confidence interval (CI) $1.11-$ 3.85; AKI-com: OR 2.53, 95\% CI 1.23-5.22].

Multivariable logistic regression analysis including known predictive factors revealed that hospital mortality among patients with stage 3 AKI-Cr increased nearly 8 -fold when compared to those without AKI-Cr (OR 7.87, 95\% CI 3.26-19.00), whereas stages 1-2 AKI-Cr were not significantly associated with an increase in hospital mortality (Table 5). When AKI-com was used instead of AKI-Cr, stage-wise increase in hospital mortality was observed and stage 2 and stage 3 AKI-com were significantly associated with an increase in hospital mortality (stage 2: OR 2.71, 95\% CI 1.14-6.46; stage 3: OR 8.66, 95\% CI 3.33-22.60; Table 5).

The addition of AKI-Cr to the known predictive factors (age, MELD score $>30$, blood-type incompatibility and re-transplantation) improved AUC-ROC for hospital mortality from 0.63 (95\% CI 0.54-0.71) to 0.71 (95\% CI 0.62-0.79); when AKI-com was used instead of AKI-Cr, the AUC-ROC was 0.74 (95\% CI 0.66-0.81) (Fig. 2). 


\section{Discussion}

To the best of our knowledge, this is the first study that investigates the incidence and the impact on clinical outcomes of oliguria in patients undergoing liver transplantation. An analysis of our cohort revealed the following: (1) oliguria meeting the urine output criterion of AKI occurred in $50.3 \%$ of LDLT recipients, (2) compared with AKI diagnosis using only SCr criteria, incorporating oliguria into the diagnostic criteria for AKI dramatically increased the number of patients diagnosed with AKI from $39.7 \%$ to $62.2 \%$, and (3) although incorporating oliguria into the diagnostic criteria for AKI only marginally improved the prognostic performance for hospital mortality, oliguria without AKI-Cr was significantly associated with prolonged ICU or hospital stays and lower CKD-free survival rate at POD 90.

The incidence, risk factors or outcomes of AKI after deceased-donor liver transplantation (DDLT) have been extensively studied, but few studies have reported them in $\operatorname{LDLT}^{23}$. The incidence of AKI-Cr after LDLT observed in this study (39.7\%) was similar to those after DDLT reported in previous studies $(39.4-56.6 \%)^{3,24,25}$. These incidences of AKI were substantially higher than those in the rest of the population who underwent major non-cardiac surgery, with a reported incidence of approximately $7.5 \%{ }^{26,27}$. Receiving a graft from a living donor had a protective effect against AKI after liver transplantation ${ }^{23}$. However, the high incidence of AKI-Cr observed in our study suggests that LDLT recipients, as well as DDLT recipients, are at extremely high risk of postoperative AKI.

Multivariable analysis revealed that severe AKI (stage 3 AKI-Cr and stage 2-3 AKI-com) was associated with substantial increase in hospital mortality. Moreover, the addition of AKI-Cr or AKI-com to the known predictive factors improved AUC-ROC for hospital mortality. These results suggest that the development of severe AKI has a significant impact on clinical outcomes after LDLT. However, we could not show significant association of stage 1 AKI-Cr or stage 1 AKI-com with increased mortality (odds ratio around 1.0). Clinical significance of small increase in SCr or temporary oliguria after liver transplantation needs to be elucidated in future studies. 
Although the aetiology of AKI after liver transplantation is multifactorial (factors include surgery-related events, blood loss, hypotension, sepsis, calcineurin inhibitor-induced vasoconstriction and volume depletion $)^{28}$, maintaining cardiovascular stability during and after liver transplantation is vital to renal perfusion and therefore is considered to be important in order to prevent AKI. In our study, patients with both AKI-Cr and AKI-UO suffered from increased blood loss compared with those without AKI diagnosed through either criterion. Similarly, although not reaching statistical significance, those with AKI-UO who also did not meet SCr-based criterion for AKI tended to have more blood loss. Hemodynamic instability due to massive blood loss may have contributed to the development of AKI.

Applying the urine output criterion in addition to the $\mathrm{SCr}$ criterion dramatically increased the number of patients diagnosed with AKI (39.7\% to 62.2\%). A similar phenomenon was observed in critically ill patients ${ }^{9,10}$ or patients undergoing cardiac surgery ${ }^{12,13}$. Because a significant proportion of patients exhibit oliguria without SCr increase, the inclusion or exclusion of the urine output criterion must result in either significant overdiagnosis or underdiagnosis of true AKI. To resolve this problem, studies are needed to elucidate the validity and utility of oliguria in diagnosing AKI. Several studies have investigated the specific role of the oliguric component of AKI definition. A study of more than 14,000 critically ill patients reported a $50-100 \%$ increase in AKI diagnosis when the urine output criterion was incorporated into the definition but with a diminished effect on mortality compared with the corresponding stage of AKI-Cr alone ${ }^{9}$. On the other hand, the additive predictive value of the oliguric component of AKI has been reported by some authors. Applying the urine output criterion in addition to the $\mathrm{SCr}$ criterion resulted in significant improvement in the predictive performance of mortality ${ }^{10}$ (AUC-ROC increased from 0.6 to 0.69 ). In patients who developed AKI, urine output alone was a better mortality predictor than $\mathrm{SCr}$ alone or the combination of both ${ }^{11}$. Additional studies have produced varying estimates for the impact of the urine output criterion on both AKI incidence and outcomes ${ }^{12,13}$. Possible explanations for the inconsistency of these results include differences in the study population. Differences in the definition of oliguria might also have 
resulted in different estimates. Some studies used modified urine output criterion, for example, using 24 hour urine output to define oliguria instead of hourly urine output ${ }^{9}$.

In the setting of advanced liver disease, recent consensus definitions for AKI recommend the use of SCr only because of the limitations of urine output in this population ${ }^{20}$. However, in our study, patients with oliguria who also did not meet SCr-based criterion for AKI had longer ICU or hospital stays and lower CKD-free survival rate at POD 90. These results suggest that oliguria without SCr increase had a significant impact on clinical outcomes after liver transplantation and discarding urine output criterion in diagnosing AKI may miss this clinically important sign.

The major limitation of this study is its retrospective design and small sample size. This study represents data from one institution. There was no standardised protocol for perioperative fluid or diuretic administration. Although hourly urine output is required to define AKI-UO according to recent guidelines, urine output measured every 2 hours was used to define oliguria instead because urine output is measured every 2 hours in our ICU. This modification may have affected the incidence or the impact on clinical outcomes of oliguria.

In conclusion, oliguria is common after liver transplantation and incorporating oliguria into the diagnostic criteria for AKI can dramatically increase the number of patients diagnosed with AKI. Compared with no AKI, AKI with oliguria without $\mathrm{SCr}$ increase was significantly associated with adverse postoperative outcomes including prolonged ICU or hospital stays and lower CKD-free survival rate at POD 90. Discarding urine output criterion in diagnosing AKI may miss this clinically important sign. 
Funding

This work was not supported by external funding.

\section{Conflicts of interest}

None. 


\section{References}

1. Barri YM, Sanchez EQ, Jennings LW, Melton LB, Hays S, Levy MF, Klintmalm GB. Acute kidney injury following liver transplantation: definition and outcome. Liver Transpl 2009; 15: 475-83

2. Ferreira AC, Nolasco F, Carvalho D, Sampaio S, Baptista A, Pessegueiro P, Monteiro E, Mourão L, Barroso E. Impact of RIFLE classification in liver transplantation. Clin Transplant 2010; 24: 394-400 3. Hilmi IA, Damian D, Al-Khafaji A, Planinsic R, Boucek C, Sakai T, Chang CC, Kellum JA. Acute kidney injury following orthotopic liver transplantation: incidence, risk factors, and effects on patient and graft outcomes. Br J Anaesth 2015; 114: 919-26

4. Bellomo R, Ronco C, Kellum JA, Mehta RL, Palevsky P; Acute Dialysis Quality Initiative workgroup. Acute renal failure - definition, outcome measures, animal models, fluid therapy and information technology needs: the Second International Consensus Conference of the Acute Dialysis Quality Initiative (ADQI) Group. Crit Care 2004; 8: R204-12

5. Mehta RL, Kellum JA, Shah SV, Molitoris BA, Ronco C, Warnock DG, Levin A; Acute Kidney Injury Network. Acute Kidney Injury Network: report of an initiative to improve outcomes in acute kidney injury. Crit Care. 2007; 11: R31

6. Kidney Disease: Improving Global Outcomes (KDIGO) Acute Kidney Injury Work Group. KDIGO clinical practice guidelines for acute kidney injury. Kidney Int Suppl 2012; 2: 1-138

7. Ali T, Khan I, Simpson W, Prescott G, Townend J, Smith W, Macleod A. Incidence and outcomes in acute kidney injury: a comprehensive population-based study. J Am Soc Nephrol 2007; 18: 1292-8

8. Ostermann M, Chang RWS. Acute kidney injury in the intensive care unit according to RIFLE. Crit Care Med 2007; 35: 1837-43

9. Joannidis M, Metnitz B, Bauer P, Schusterschitz N, Moreno R, Druml W, Metnitz PG. Acute kidney injury in critically ill patients classified by AKIN versus RIFLE using the SAPS 3 database. Intensive Care Med 2009; 35: 1692-702

10. Macedo E, Malhotra R, Bouchard J, Wynn SK, Mehta RL. Oliguria is an early predictor of higher mortality in critically ill patients. Kidney Int 2011; 80: 760-7 
11. Mandelbaum T, Scott DJ, Lee J, Mark RG, Malhotra A, Waikar SS, Howell MD, Talmor D. Outcome of critically ill patients with acute kidney injury using the Acute Kidney Injury Network criteria. Crit Care Med 2011; 39: 2659-64

12. Haase M, Bellomo R, Matalanis G, Calzavacca P, Dragun D, Haase-Fielitz A. A comparison of the RIFLE and Acute Kidney Injury Network classifications for cardiac surgery-associated acute kidney injury: a prospective cohort study. J Thorac Cardiovasc Surg 2009; 138: 1370-6

13. McIlroy DR, Argenziano M, Farkas D, Umann T, Sladen RN. Incorporating oliguria into the diagnostic criteria for acute kidney injury after on-pump cardiac surgery: impact on incidence and outcomes. J Cardiothorac Vasc Anesth 2013; 27: 1145-52

14. Matsuo S, Imai E, Horio M, Yasuda Y, Tomita K, Nitta K, Yamagata K, Tomino Y, Yokoyama H, Hishida A; Collaborators developing the Japanese equation for estimated GFR. Revised equations for estimated GFR from serum creatinine in Japan. Am J Kidney Dis 2009; 53: 982-92

15. Wiesner R, Edwards E, Freeman R, Harper A, Kim R, Kamath P, Kremers W, Lake J, Howard T, Merion RM, Wolfe RA, Krom R; United Network for Organ Sharing Liver Disease Severity Score Committee. Model for end-stage liver disease (MELD) and allocation of donor livers. Gastroenterology 2003; 124: 91-6

16. Inomata $\mathrm{Y}$, Tanaka K, Egawa H, Uemoto S, Ozaki N, Okajima H, Satomura K, Kiuchi T, Yamaoka Y, Hashida T. The evolution of immunosuppression with FK506 in pediatric living-related liver transplantation. Transplantation 1996; 61: 247-52

17. Egawa H, Ohmori K, Haga H, Tsuji H, Yurugi K, Miyagawa-Hayashino A, Oike F, Fukuda A, Yoshizawa J, Takada Y, Tanaka K, Maekawa T, Ozawa K, Uemoto S. B-cell surface marker analysis for improvement of rituximab prophylaxis in ABO-incompatible adult living donor liver transplantation. Liver Transpl 2007; 13: 579-88

18. Egawa H, Ohdan H, Haga H, Tsuruyama T, Oike F, Uemoto S, Ozawa K. Current status of liver transplantation across ABO blood-type barrier. J Hepatobiliary Pancreat Surg 2008; 15: 131-8 
19. Egawa H, Teramukai S, Haga H, Tanabe M, Fukushima M, Shimazu M. Present status of ABO-incompatible living donor liver transplantation in Japan. Hepatology 2008; 47: 143-52

20. Wong F, Nadim MK, Kellum JA, Salerno F, Bellomo R, Gerbes A, Angeli P, Moreau R, Davenport A, Jalan R, Ronco C, Genyk Y, Arroyo V. Working Party proposal for a revised classification system of renal dysfunction in patients with cirrhosis. Gut 2011; 60: 702-9

21. Rana A, Hardy MA, Halazun KJ, Woodland DC, Ratner LE, Samstein B, Guarrera JV, Brown RS Jr, Emond JC. Survival outcomes following liver transplantation (SOFT) score: a novel method to predict patient survival following liver transplantation. Am J Transplant 2008; 8: 2537-46

22. Kaido T, Egawa H, Tsuji H, Ashihara E, Maekawa T, Uemoto S. In-hospital mortality in adult recipients of living donor liver transplantation: experience of 576 consecutive cases at a single center. Liver Transpl 2009; 15: 1420-5

23. Hilmi IA, Damian D, Al-Khafaji A, Sakai T, Donaldson J, Winger DG, Kellum JA. Acute kidney injury after orthotopic liver transplantation using living donor versus deceased donor grafts: A propensity score-matched analysis. Liver Transpl 2015; 21: 1179-85

24. Klaus F, Keitel da Silva C, Meinerz G, Carvalho LM, Goldani JC, Cantisani G, Zanotelli ML, Duro Garcia V, Keitel E. Acute kidney injury after liver transplantation: incidence and mortality. Transplant Proc 2014; 46: 1819-21

25. Karapanagiotou A, Dimitriadis C, Papadopoulos S, Kydona C, Kefsenidis S, Papanikolaou V, Gritsi-Gerogianni N. Comparison of RIFLE and AKIN criteria in the evaluation of the frequency of acute kidney injury in post-liver transplantation patients. Transplant Proc 2014; 46: 3222-7

26. Abelha FJ, Botelho M, Fernandes V, Barros H. Determinants of postoperative acute kidney injury. Crit Care 2009; 13: R79

27. Thakar CV. Perioperative acute kidney injury. Adv Chronic Kidney Dis 2013; 20: 67-75

28. Bilbao I, Charco R, Balsells J, Lazaro JL, Hidalgo E, Llopart L, Murio E, Margarit C. Risk factors for acute renal failure requiring dialysis after liver transplantation. Clin Transplant 1998; 12: 123-9 
Tables

Table 1 Definition and staging for acute kidney injury ${ }^{6}$.

\begin{tabular}{lll}
\hline Stage & Serum creatinine & Urine output \\
\hline 1 & $1.5-1.9$ times baseline or $\geq 0.3 \mathrm{mg} / \mathrm{dl}$ increase & $<0.5 \mathrm{~mL} / \mathrm{kg} / \mathrm{h}$ for $6-12 \mathrm{~h}$ \\
2 & $2.0-2.9$ times baseline & $<0.5 \mathrm{~mL} / \mathrm{kg} / \mathrm{h} \mathrm{for} \geq 12 \mathrm{~h}$ \\
3 & 3.0 times baseline or increase to $4.0 \mathrm{mg} / \mathrm{dl}$ or $\quad<0.3 \mathrm{~mL} / \mathrm{kg} / \mathrm{h}$ for $\geq 24 \mathrm{~h}$ or anuria \\
& initiation of renal replacement therapy & for $\geq 12 \mathrm{~h}$ \\
\hline
\end{tabular}




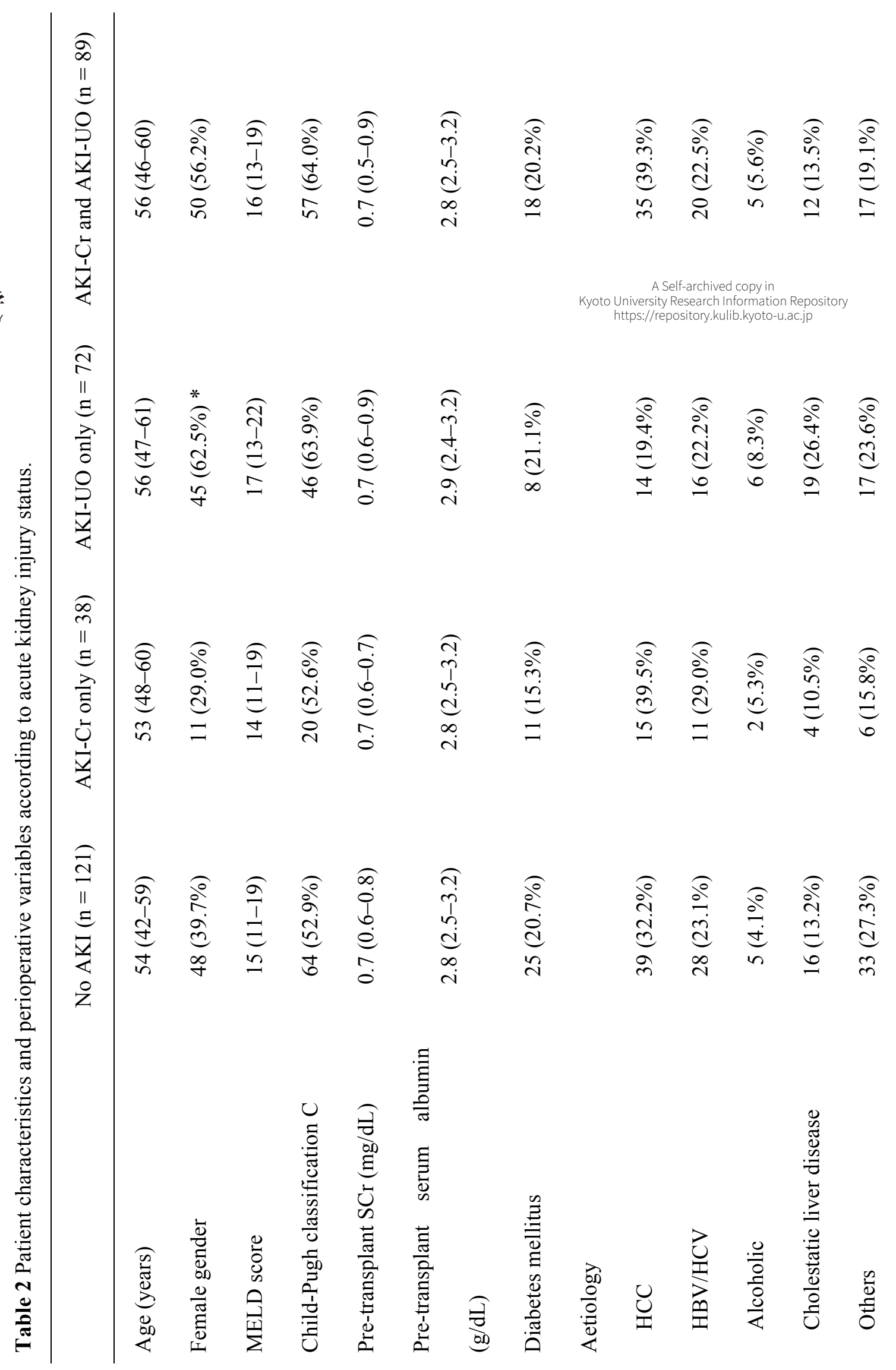




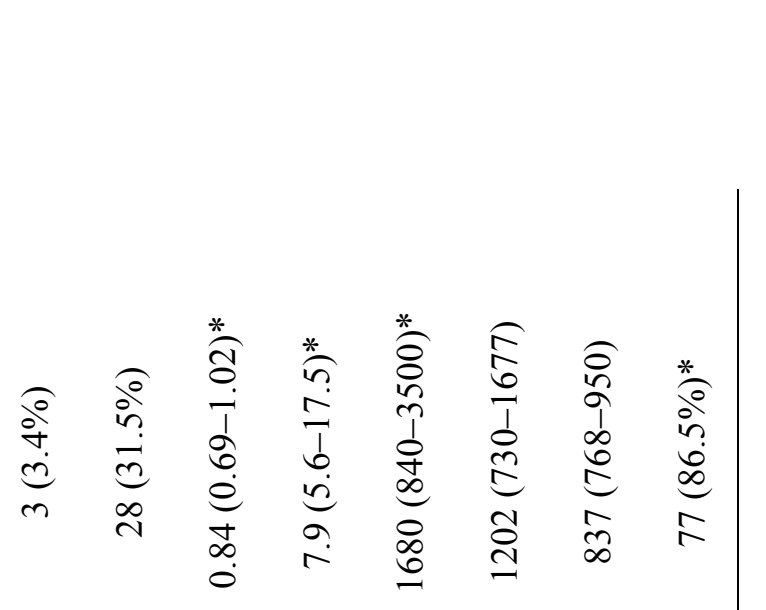

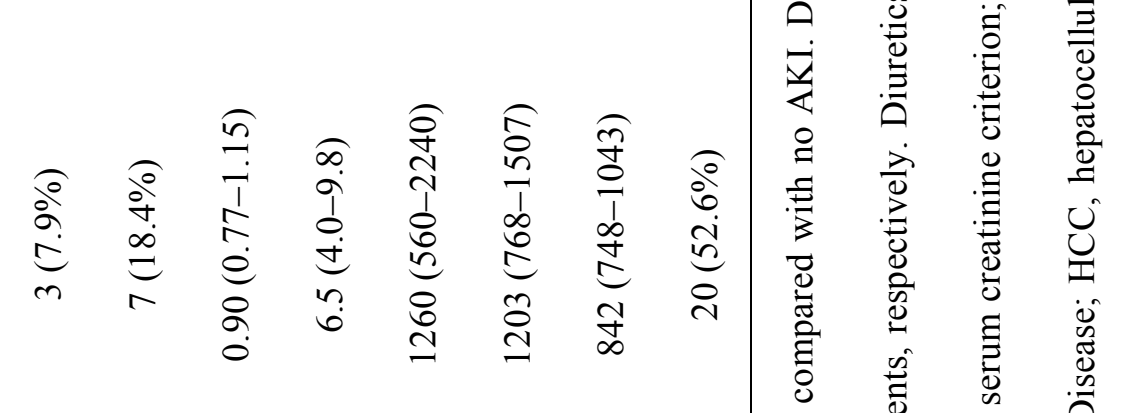

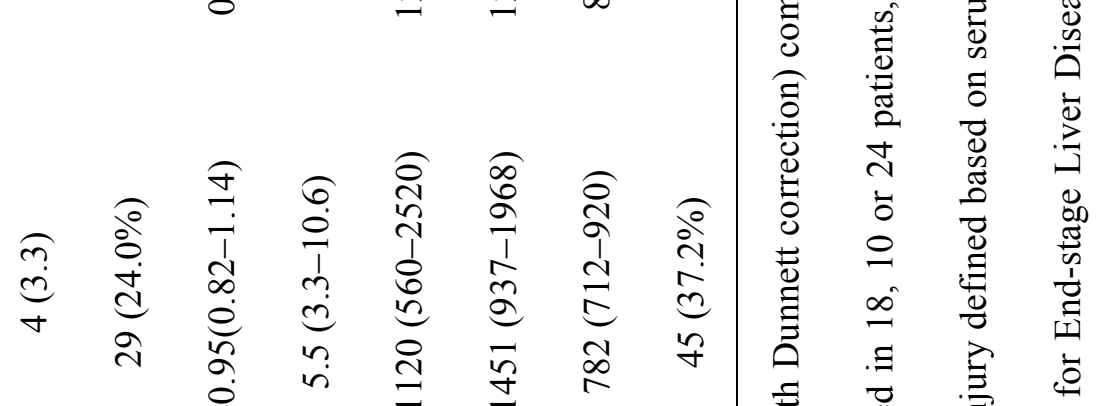

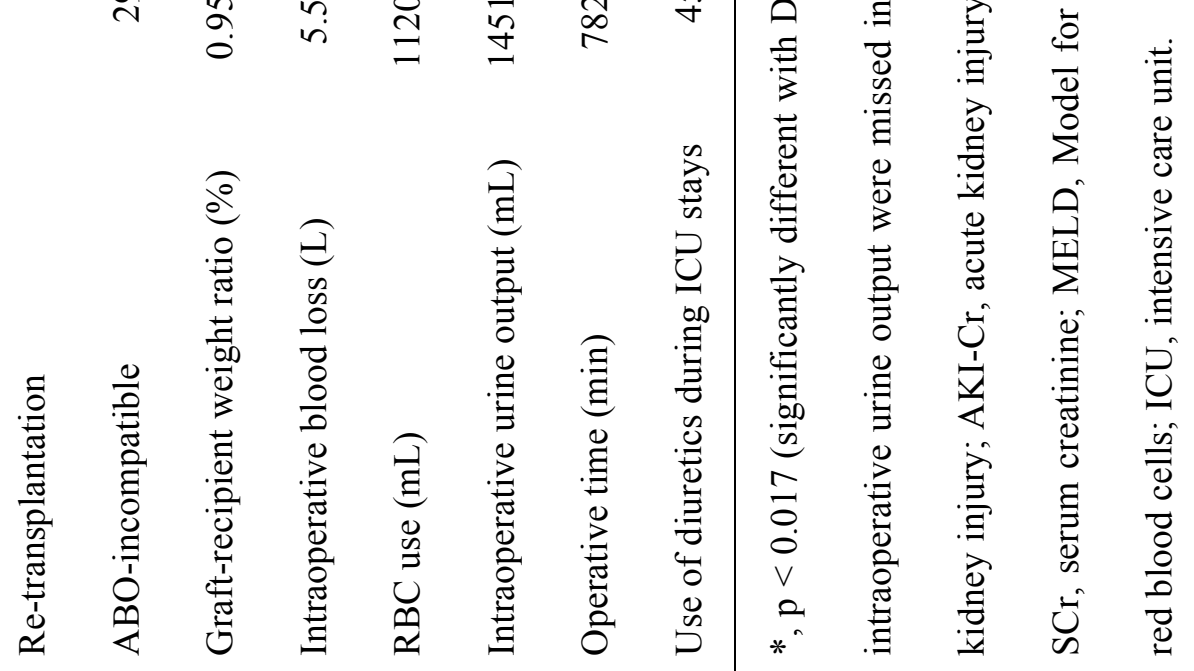


$\stackrel{9}{\circ}$

号
0
0
0
0
0
0
0
0
0
0
0
0
0
0
0
0
0

(3.).京都大学

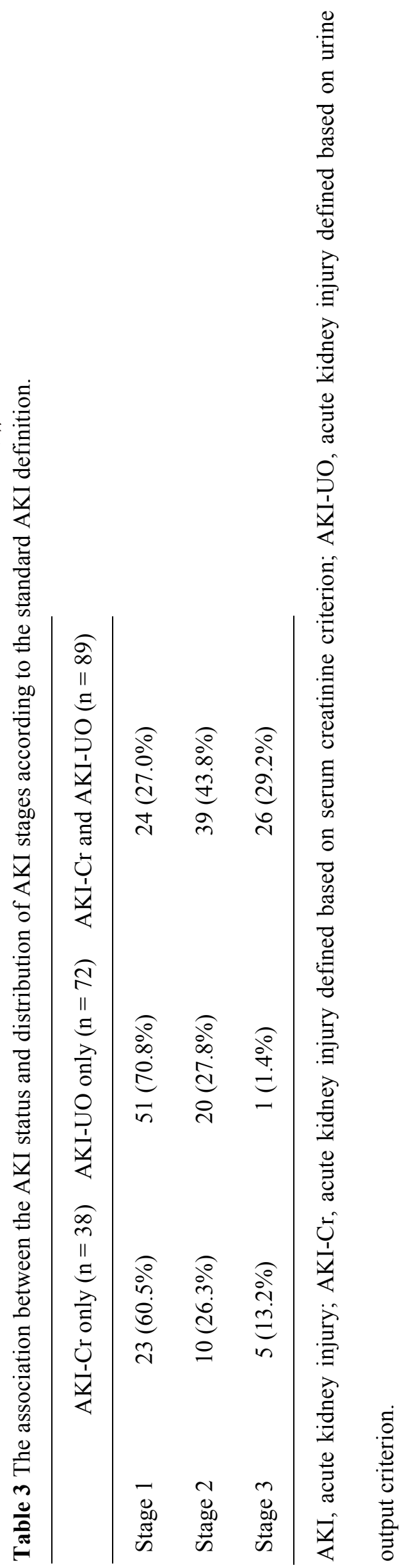


จำ

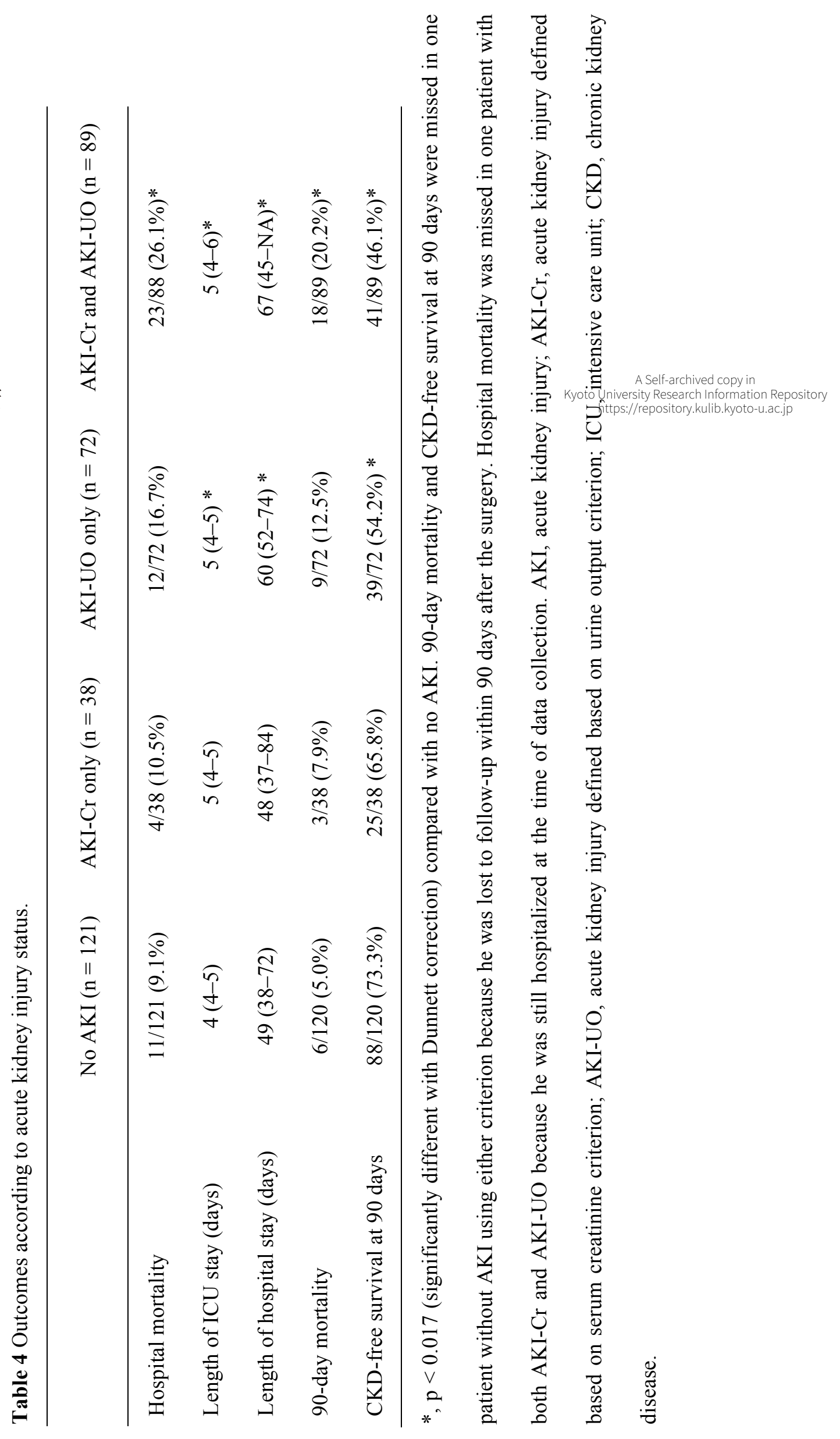




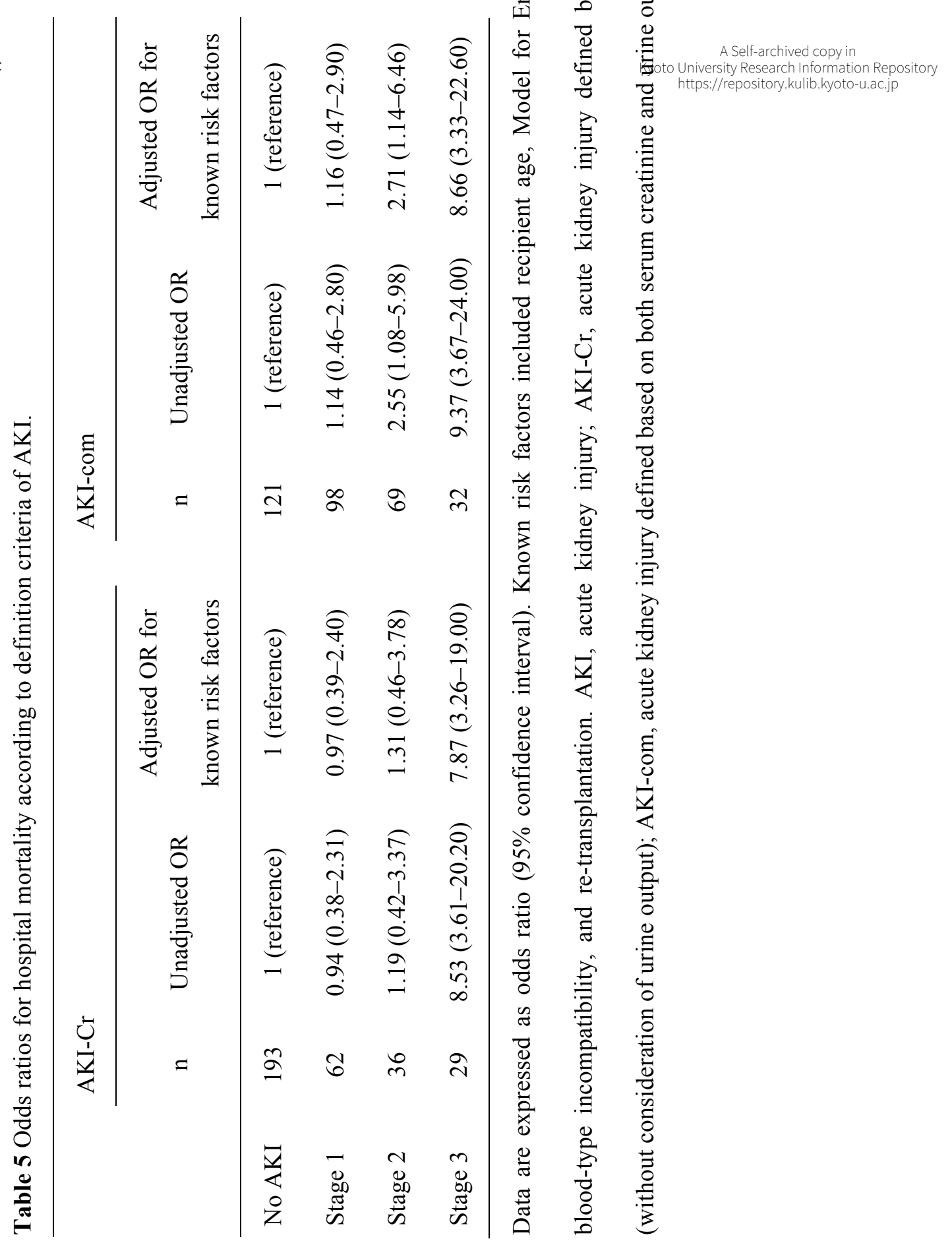


Figure legends

Fig. 1. Venn diagram of AKI status. AKI, acute kidney injury; AKI-Cr, acute kidney injury defined based on serum creatinine criterion; and AKI-UO, acute kidney injury defined based on urine output criterion.

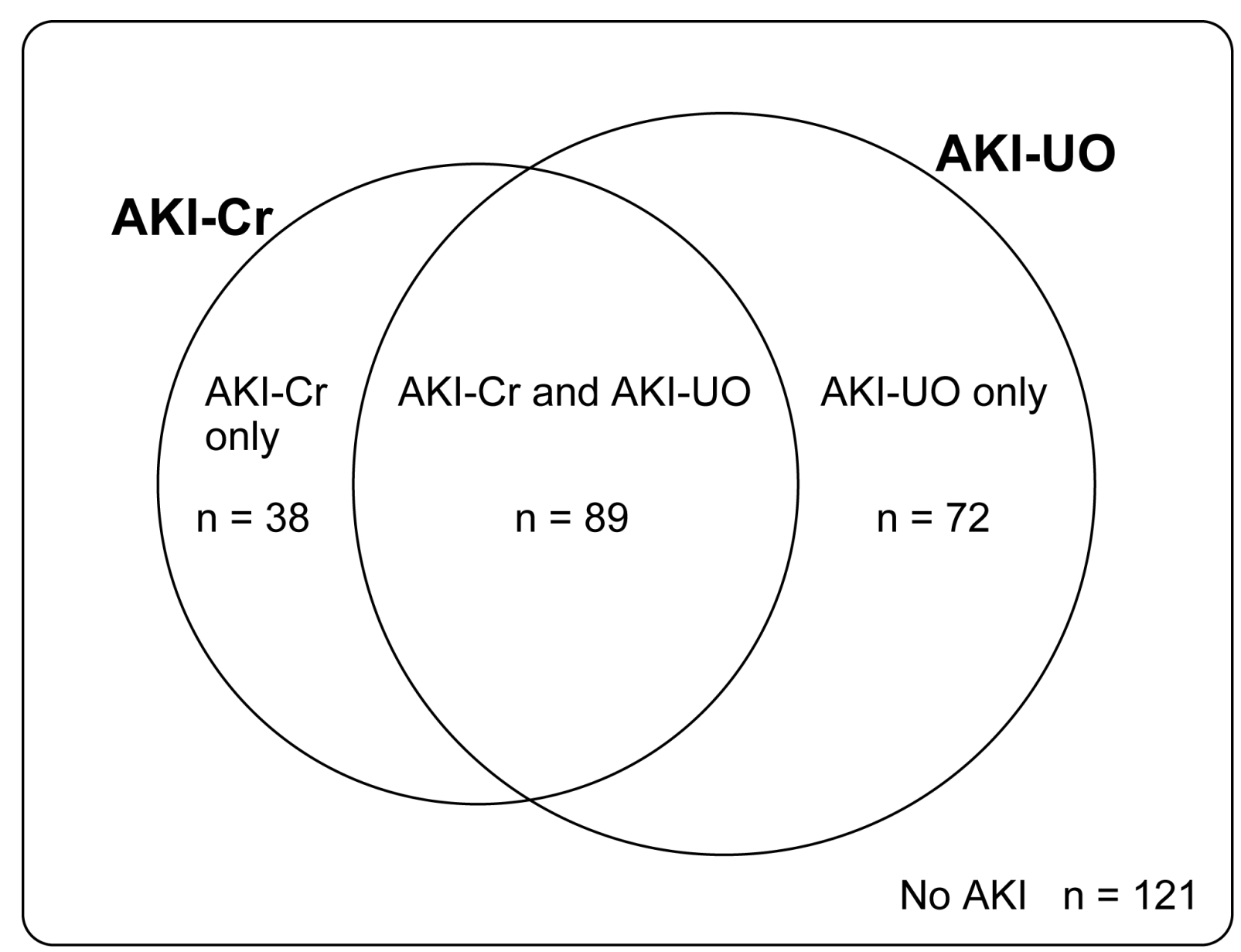

Fig. 2. The areas under the receiver operating characteristic curves for hospital mortality as predicted by known risk factors only, AKI-Cr combined with known risk factors and AKI-com combined with known risk factors. Known risk factors included recipient age, Model for End-stage Liver Disease score $>30$, blood type incompatibility and re-transplantation. The AUC values and 95\% CIs are listed within the figure. AUC, area under the curve; CI, confidence interval; AKI-Cr, acute kidney injury 
defined based on serum creatinine criterion (without consideration of urine output); AKI-com, acute kidney injury defined based on both serum creatinine and urine output criteria.

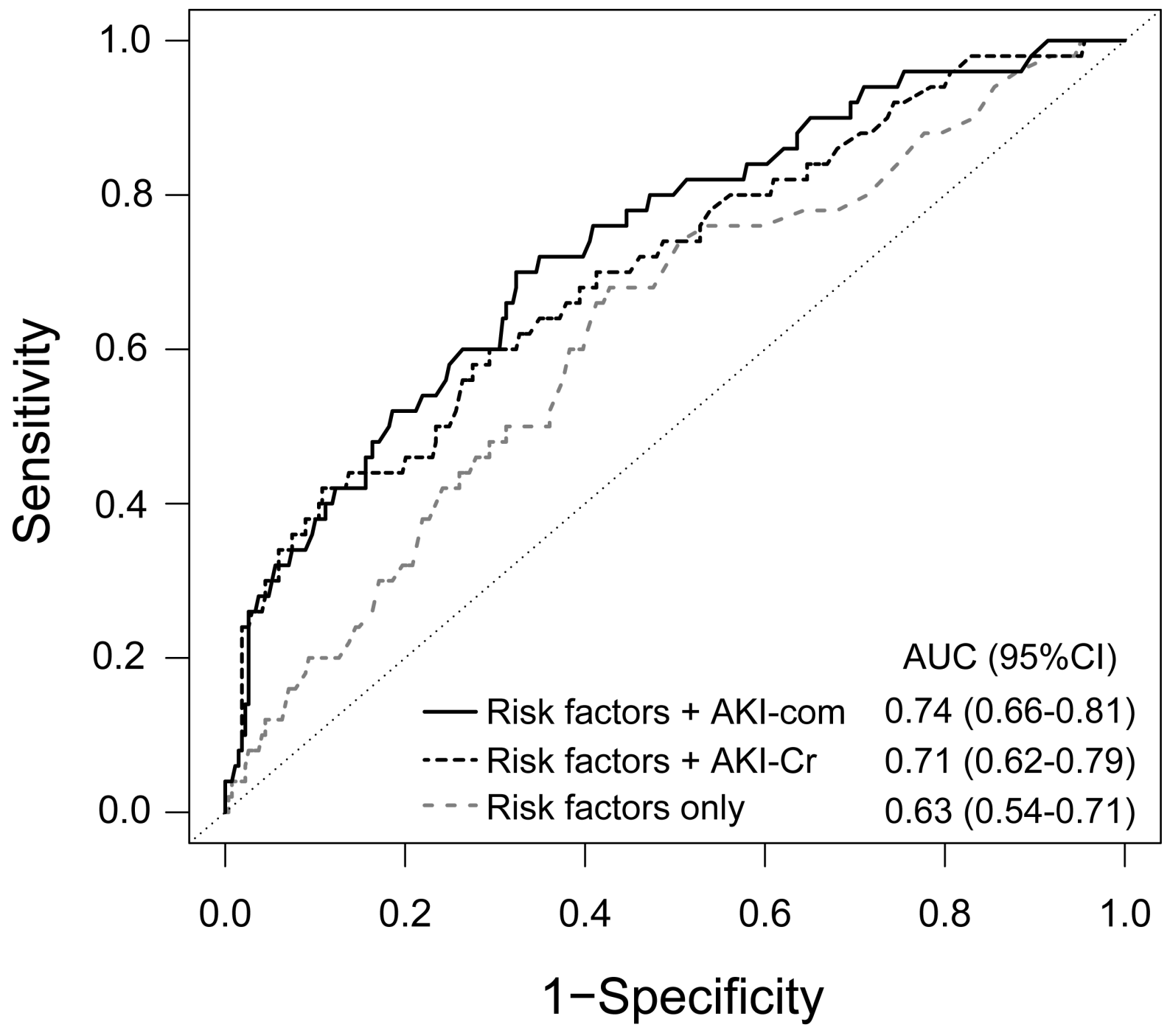

